\title{
Design and analysis of monolithic triple-stacked power amplifiers using GaAs HBT-HEMT process
}

\author{
Chih-Chun Shen ${ }^{1}$, Wei-Cheng Chen $^{1}$, and Hong-Yeh Chang ${ }^{1 \text {, a) }}$
}

Abstract This paper describes design and analysis of monolithic triplestacked power amplifiers (PAs) in $2-\mu \mathrm{m} / 0.5-\mu \mathrm{m}$ GaAs HBT-HEMT process. The proposed PAs are designed using both heterojunction bipolar transistor (HBT) and high electron-mobility transistor (HEMT). Based on a common-emitter (CE) configuration of HBT with stacked common-gate (CG) configuration of HEMTs or stacked common-base (CB) configuration of HBT as the third-stacked transistor, better power performances with good PAEs can be achieved as compared with the CE or common-source (CS) amplifier due to high output stacking impedance. The bandwidth with HEMT/CG configuration as the third-stacked transistor is investigated. The proposed stacked PAs demonstrate a maximum output powers of $31.7 \mathrm{dBm}$, a compact chip size of within $1.6 \times 1 \mathrm{~mm}^{2}$, and a maximum power added efficiency (PAE) of $38.3 \%$ at $5 \mathrm{GHz}$.

Keywords: GaAs, heterojunction bipolar transistor (HBT), high electronmobility transistor (HEMT), microwave circuits, monolithic microwave integrated circuit (MMIC), power amplifier (PA)

Classification: Microwave and millimeter-wave devices, circuits, and modules

\section{Introduction}

Stacked transistor is widely used in several radio frequency (RF) circuits. Based on the voltage multiplication, the output power can be enhanced with high output impedance for the power amplifier (PA) design [1]. For the conventional stacked PA, a common-source (CS) transistor is used to drive a common-gate (CG) transistor. With a proper gate termination, the output power can be enhanced by the maximum power of each transistor. For the conventional $n$-stacked PA, the stacked transistors are all designed using the same type transistor, such as $\operatorname{HBT}[2,3,4,5,6,7,8,9,10]$, NMOS $[11,12,13,14,15,16,17,18]$ and high electronmobility transistor (HEMT) [19, 20, 21, 22]. A broadband monolithic GaAs heterojunction bipolar transistor (HBT)-HEMT/common-emitter (CE) and common-gate (CG) stacked PA has been proposed in [23]. Based on a CE transistor stacked with a CG transistor, the wide bandwidth and high output power can be achieved. The monolithic HBT-HEMT process offers great design flexibility to achieve superior performance. To date, a frequency quadrupler [24], distributed amplifiers [25, 26], a frequency tripler [27], voltage-controlled oscillators [28, 29], and a variable gain

${ }^{1}$ Dept. of Electrical Engineering, National Central University, Jhongli District, Taoyuan, 32001, Taiwan.

a) hychang@ee.ncu.edu.tw

DOI: $10.1587 /$ elex.17.20200172

Received May 13, 2020

Accepted May 28, 2020

Publicized June 10, 2020

Copyedited July 10, 2020 amplifier [30] have been presented using several advanced HBT-HEMT processes.

In this paper, two PAs are designed using a triple-stacked topology. Based on the CE-CG configuration with two different stacked transistors, the proposed triple-stacked PAs perform watt-level high output power. The device selection and optimal capacitance of gate terminal capacitor are presented. For broadband and high output power performance, the triple-stacked PA using CE-CG-CG (Type-I) configuration with stacked depletion-mode (D-mode) HEMT is used. The input and output matching networks are also designed to achieve broad bandwidth. The proposed PAs feature a maximum output powers of higher than $30 \mathrm{dBm}$, a maximum power added efficiency (PAE) of $30 \%$, and a compact chip size of within $1.6 \times 1 \mathrm{~mm}^{2}$. As compared to the PA in [23], the proposed stacked PAs are designed using CECG-CG (Type-I) and CE-CG-CB (Type-II) triple-stacked topologies. The Type-II PA is designed to compare with the Type-I PA. The bandwidth and output power are investigated. The design methodology of triple-stacked PAs using the HBT-HEMT process is presented with theoretical calculation. With a stacked CG HEMT transistor in triplestacked PA, the higher output power can be achieved with good efficiency.

\section{Stacked transistor selection and capacitance design}

The proposed triple-stack PAs are designed using the GaAs HBT-HEMT process provided by the WIN Semiconductors Corporation, Taiwan. The breakdown voltages of the HBT and HEMT are 15 and $20 \mathrm{~V}$, respectively. The unity current gain frequencies $\left(f_{T}\right)$ are higher than 35 and $40 \mathrm{GHz}$ for HEMT and HBT, respectively. The maximum oscillation frequencies $\left(f_{\max }\right)$ are higher than 100 and $70 \mathrm{GHz}$ for HEMT and HBT, respectively. The schematics of the proposed triple-stacked PAs are shown in Fig. 1. The triplestacked PA is composed of one CE transistor with two stacked CG (or CB) transistors. The output power of $n$ stacked PA can be expressed as [23]

$$
P_{\text {out }}=n^{2} \frac{\left(V_{d s}-V_{k}\right)^{2}}{2 R_{\text {opt }}}=\frac{n}{2} I_{d}^{2} R_{L},
$$

where $n$ is 3 for our proposed PAs, $R_{o p t}, R_{L}, V_{d s}, V_{k}$, and $I_{d}$ are the optimum output load impedance, load impedance of stacked transistor, drain-to-source voltage, knee voltage, and dc supply current, respectively. $R_{o p t}$ is

$$
R_{\text {opt_ } P A}=n R_{L}=n \frac{\Delta V}{\Delta I}=n \frac{V_{d s}-V_{k}}{I_{d}}
$$




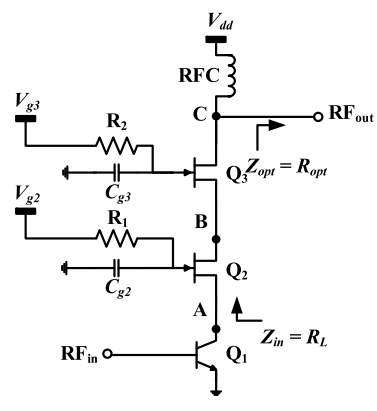

(a)

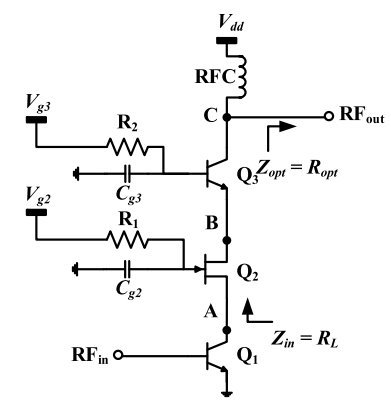

(b)
Fig. 1 Schematics of the proposed (a) Type-I, and (b) Type-II triplestacked PAs.

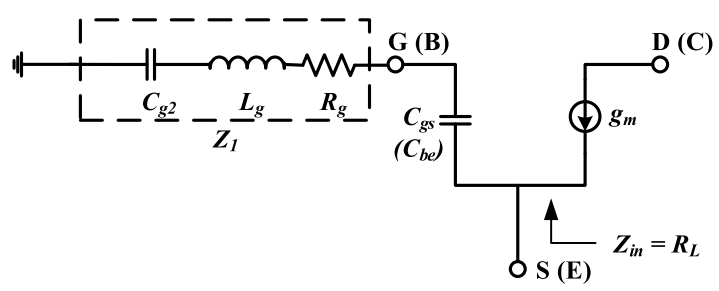

Fig. 2 Equivalent CG (or CB) circuit of the stacked transistor.

Table I Device characteristics of the transistors.

\begin{tabular}{|c|c|c|c|c|c|c|}
\hline Device & $\begin{array}{c}g_{m} \\
(\mathrm{~S})\end{array}$ & $\begin{array}{c}C_{b e}\left(C_{g s}\right) \\
(\mathrm{pF})\end{array}$ & $\begin{array}{c}R_{L} \\
(\Omega)\end{array}$ & $\begin{array}{c}V_{k} \\
(\mathrm{~V})\end{array}$ & $\begin{array}{c}C_{g 3} \\
(\mathrm{pF})\end{array}$ & $Z_{i n}$ \\
\hline HBT & 0.082 & 0.28 & 17.5 & 0.57 & 0.6 & 17.9 \\
\hline $\begin{array}{c}\text { D-mode } \\
\text { HEMT }\end{array}$ & 0.151 & 1.2 & 16.3 & 0.81 & 0.74 & 17.4 \\
\hline $\begin{array}{c}\text { E-mode } \\
\text { HEMT }\end{array}$ & 0.291 & 1.4 & 15.1 & 1.04 & 0.4 & 15.5 \\
\hline
\end{tabular}

where $R_{L}$ is the load impedance of each stacked transistor. From (2), $R_{\text {opt_PA }}$ increases with $R_{L}$. Since the dc currents (Id) across each transistor of the stacked PA are all the same, the output power can be enhanced by stacking the transistor. The optimum output impedance has been addressed in [23]. The input impedance looked into the source of the equivalent circuit in Fig. 2 can be expressed as

$$
Z_{\text {in }}=\frac{1}{g_{m}}\left[1+\frac{C_{g s}}{C_{g 2}}-\omega^{2} L_{g} C_{g s}+j \omega R_{g} C_{g s}\right]
$$

where $g_{m}, C_{g s}\left(C_{b e}\right)$, and $C_{g 2}$ are the transconductance, gate-to-source (base-to-emitter) capacitance, and gate termination capacitance, respectively, for $Q_{2}$ in Fig. $1 . R_{g}$ and $L_{g}$ are parasitic resistance and inductance. The maximum output power can be estimated using (1) and (2) from $V_{d s}$, $V_{k}$, and $R_{L}$ of each transistor. The device characteristics of the HBT-HEMT process, including HBT, E/D-mode HEMTs, are summarized in Table I with a $V_{d s}\left(\right.$ or $\left.V_{c e}\right)$ of $4 \mathrm{~V}$. Both stacked PAs are with a dc supply voltage $\left(V_{d d}\right)$ of $12 \mathrm{~V}$, and an $I_{d}$ of $196 \mathrm{~mA}$. The $V_{k}$, and $R_{L}$ of each transistor are shown in Table I. The simulated current is $196 \mathrm{~mA}$ to achieve the maximum output power of $30 \mathrm{dBm}$. The maximum output power and the increment of output power versus stacked number $(n)$ are shown in Fig. 3. As compared to the conventional CE (or CS) amplifier, the output power of the proposed stacked PA with a stacked number $(n)$ of 2 has the

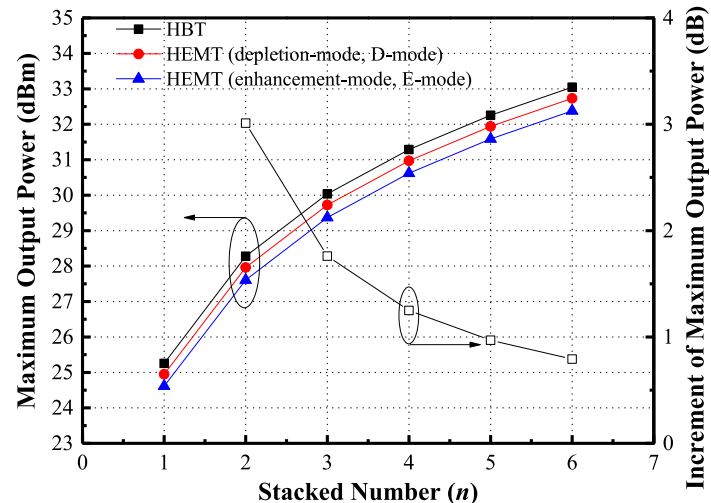

Fig. 3 Simulated maximum output power and increment of maximum output power versus stacked number $(n)$.

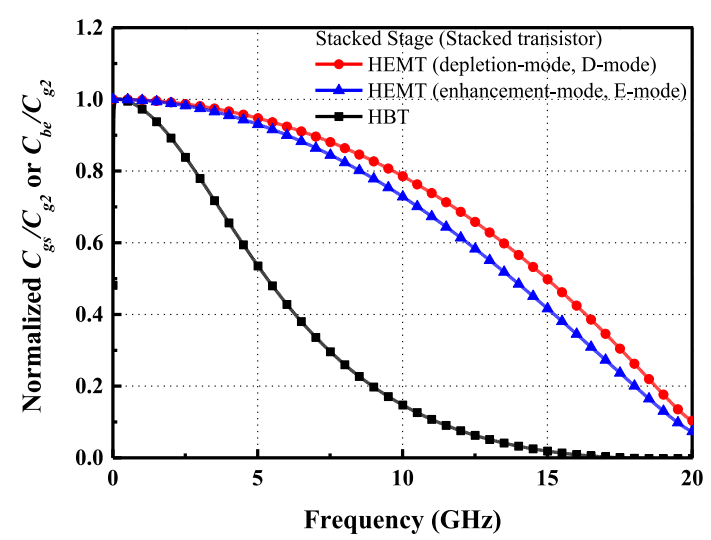

Fig. 4 Simulated normalized capacitance ratio of $C_{g s} / C_{g 2}$ and $C_{b e} / C_{g 2}$ versus frequency.

best improvement of output power up to $3 \mathrm{~dB}$. The output power increases as the stacked number $(n)$ increases, but the improvement of output power decreases. To have the best stacked efficiency with large output power, $n$ is 3 for the proposed stacked PAs.

From (3), the input impedance $\left(Z_{i n}\right)$ of the stacked transistor is varying with the capacitance ratio of $C_{g s} / C_{g 2}$, and the phase of the input impedance $\left(Z_{i n}\right)$ is also affected by the capacitance ratio. In order to achieve broad bandwidth, the matching between the $R_{o p t}$ and $R_{L}$ should be properly designed. Since the $C_{g s} / C_{b e}$ of the simple equivalent circuit in Fig. 2 is varying with the dc bias, RF power, and frequency, the impedance, $Z_{i n}$, is affected. The output bandwidth of the stacked PA is affected by the phase of $Z_{i n}$. The simulated normalized capacitance ratio $C_{g s} / C_{g 2}$ or $C_{b e} / C_{g 2}$ versus frequency is plotted in Fig. 4. The simulated normalized capacitance ratio $C_{g s} / C_{g 2}$ for the stacked transistor using depletion-mode (D-mode) HEMT features the widest frequency response, and therefore the stacked PA using the HBT-HEMT(D)/CE-CG configuration has potential to demonstrate the broad bandwidth.

To enhance the maximum output power with good efficiency, the triple-stacked PAs are designed and implemented. For the simulated results, the capacitance of $C_{g 2}$ and $C_{g 3}$ should be properly selected based on the dynamic load lines of $Q_{2}$ and $Q_{3}$. To achieve the maximum output power, the stacked transistors $\left(Q_{2}\right.$ and $\left.Q_{3}\right)$ should have the largest voltage swing between the drain and the source. The 


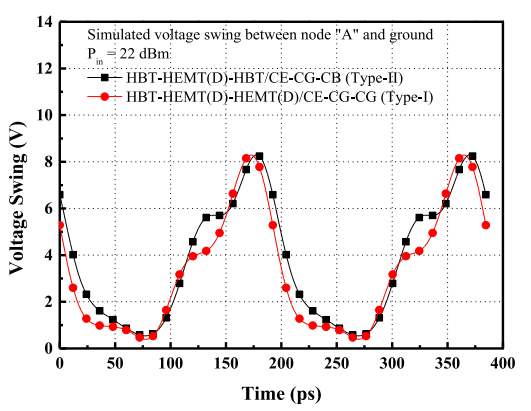

(a)

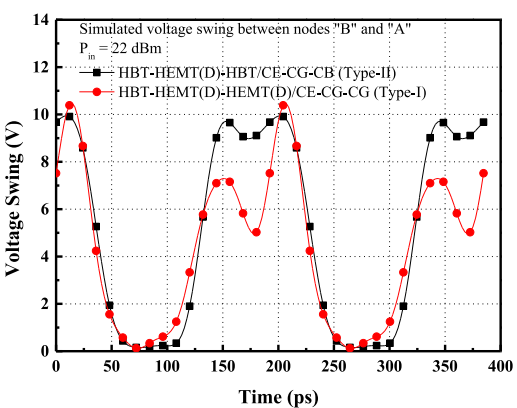

(b)

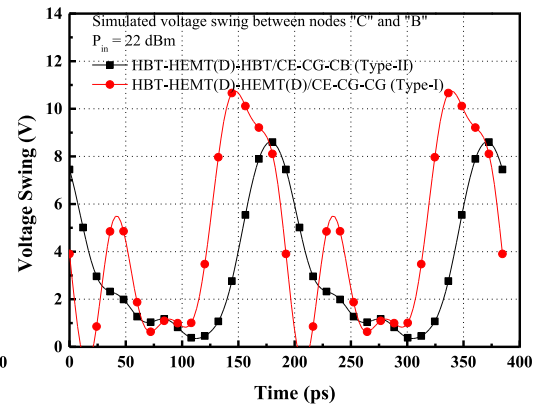

(c)

Fig. 5 The simulated voltage swings for the Type-I and Type-II configurations: (a) between node "A" and ground with an input power of $22 \mathrm{dBm}$, (b) between nodes "B" and "A" with an input power of $22 \mathrm{dBm}$, (c) between nodes "C" and "B" with an input power of $22 \mathrm{dBm}$.

large voltage swings of the PAs can be achieved with proper capacitances of $C_{g 2}$ and $C_{g 3}$, as well as the optimum load impedance. In Fig. 2, $C_{g}$ performs a capacitive voltage divider and produces the proper in-phase voltage swing at the gate and drain. For the triple-stacked PA design, the most critical parameter is the capacitance of $C_{g 2}$ and $C_{g 3}$ due to the output power. Moreover, the HEMT is more suitable for the upper transistor of the stacked PA since the HEMT has larger breakdown voltage than the HBT (higher than $5 \mathrm{~V}$ ). The voltage swing across nodes "B" and "A" in Fig. 1 is affected by the third-stacked transistor. As compared to the enhancement-mode (E-mode) HEMT, the D-mode is more suitable for the stacked transistor, since the knee voltage of the D-mode HEMT is lower than the E-mode HEMT and results in larger voltage swing.

Based on the HBT-HEMT(D)/CE-CG dual-stacked PA performance, the third-stacked transistor of triple-stacked PA is designed to enhance the output power with high efficiency and broad bandwidth. The simulated voltage swings between node " $A$ " and ground, between nodes " $B$ " and " $A$ ", and between nodes " $C$ " and "B" in Fig. 1 are plotted in Fig. 5 for the HBT-HEMT(D)-HEMT(D)/CE-CG-CG (Type-I) and HBT-HEMT(D)-HBT/CE-CG-CB (Type-II) configurations. To make fair comparison between the two PAs, the input powers are $22 \mathrm{dBm}$ in Fig. 5. As can be observed, the voltage swing across the second-stacked transistor of the Type-I configuration is higher than the Type-II configuration. Although the voltage swing at the second-stacked transistor of the Type-II configuration is higher than the Type-I for most of the period, the voltage swings at the third-stacked transistor of the Type-I configuration is much higher than the Type-II as shown in Fig. 5(b) and (c). In addition, the gate dc bias of the stacked transistor can also be lowered since the gate-to-source voltage of the D-mode HEMT is negative. The simulated insertion phases between nodes " $C$ " and "B" for the stacked HBT and HEMT transistors with the gate capacitances are shown in Fig. 6. As can be observed, the stacked transistor of HEMT(D) has wider frequency response than the HBT. Therefore, the Type-I configuration is more suitable for the triple-stacked PA design in the GaAs HBT-HEMT technology due to its high maximum output power and wide bandwidth performance.

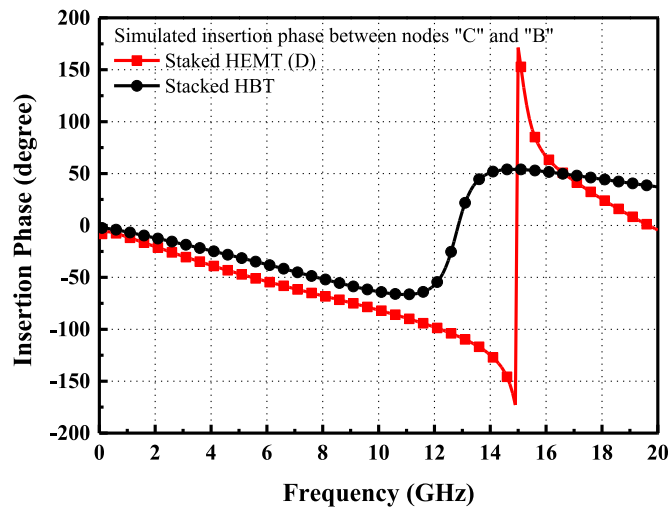

Fig. 6 The simulated insertion phases between nodes " $C$ " and "B" in Fig. 1 for the Type-I (stacked HEMT) and Type-II (stacked HBT) configurations.

\section{Circuit design and power amplifier implementation}

To verify the proposed design concept, two stacked PAs are implemented using GaAs HBT-HEMT process. The schematics of the proposed triple-stacked PAs are shown in Fig. 7. Since the $n$-stacked amplifier has $n$ times of the load impedance $R_{L}$, the output impedance of the stacked amplifier should be close to the system impedance $\left(Z_{0}\right)$ as compared to the conventional CS (or CE) amplifier, especially for the large device size. Therefore, the output matching network can be designed using 2-section LC network in the region of low Constant- $Q\left(Q_{n}\right)$ contour to achieve broad bandwidth. The termination networks $\mathrm{C}_{g 2}$, and $C_{g 3}$ are designed using (3). The emitter area of $Q_{1}$ is $1080 \mu \mathrm{m}^{2}$, and the total gate width of $Q_{2}$ is $2400 \mu \mathrm{m}$. Since the overall current of the stacked PA is limited by the $Q_{1}$, the sizes of the thirdtransistors are chosen to be the same as $Q_{1}$ and $Q_{2}$ to make sure that $Q_{3}, Q_{2}$, and $Q_{1}$ have the same dc performance. For the Type-I triple-stacked PA, the total gate width of $Q_{3}$ is $2400 \mu \mathrm{m}$. For the Type-II triple-stacked PA, the emitter area of $Q_{3}$ is $1080 \mu \mathrm{m}^{2}$. The device size selection and $R_{o p t}$ is simulated using load-pull setup. In order to match a 50$\Omega$ load to the optimum load impedance $R_{o p t}, 2$-section LC elements $\left(L_{5}, L_{6}, C_{5}\right.$, and $\left.C_{6}\right)$ are designed for the output matching network with a $Q_{n}$ of within 1 for the Type-I configuration. Since the $R_{\text {opt }}$ of Type-II configuration is higher than the Type-I configuration, 1-section LC elements $\left(L_{5}, L_{6}\right.$, and $\left.C_{6}\right)$ are designed for the output matching. For 


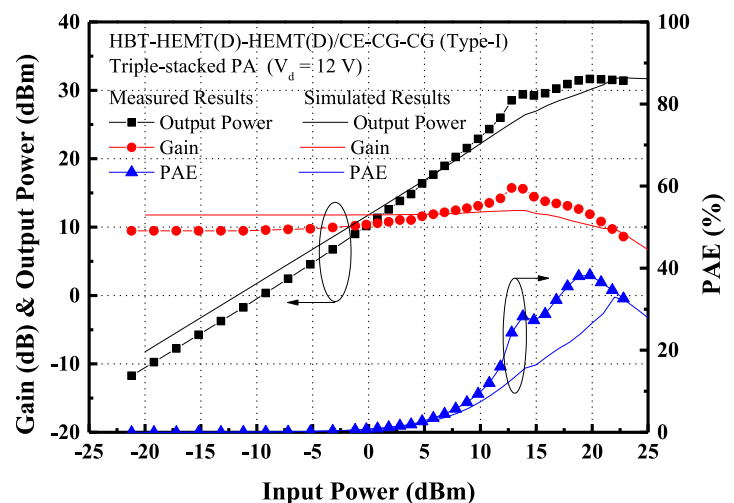

(a)

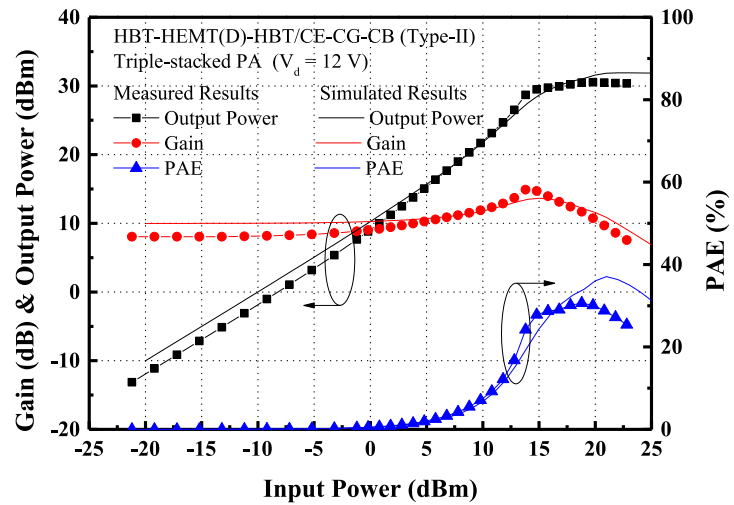

(b)

Fig. 10 Simulated and measured results at $5 \mathrm{GHz}$ : (a) output powers, gains, and PAEs for the Type-I triple-stacked PA, and (b) output powers, gains, and PAEs for the Type-II triple-stacked PAs.

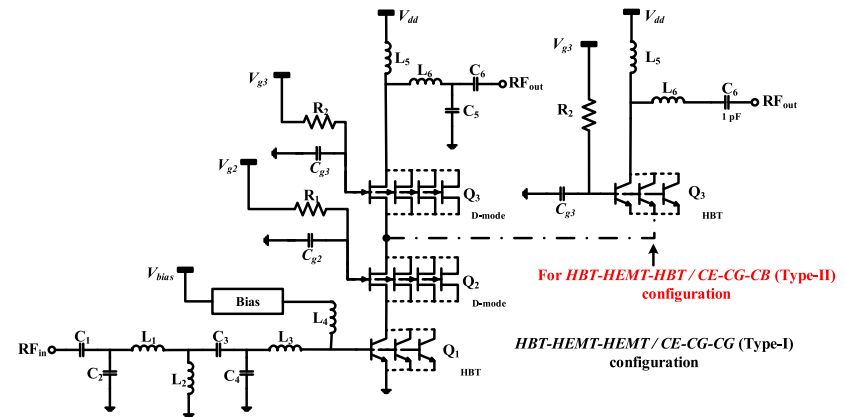

Fig. 7 The schematics of the proposed Type-I and Type-II triple-stacked PAs.

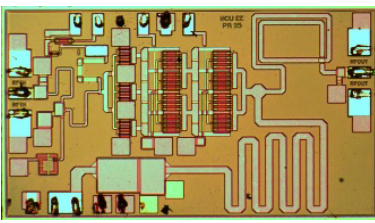

(a)

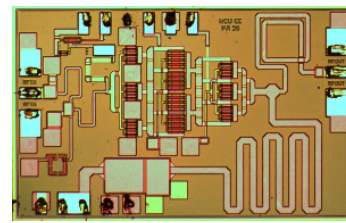

(b)
Fig. 8 Chip photos of the (a) Type-I, and (b) Type-II triple-stacked PAs with chip sizes of $1.6 \times 1 \mathrm{~mm}^{2}$ and $1.5 \times 1 \mathrm{~mm}^{2}$.

the input matching network, the 3-section LC elements $\left(L_{1}\right.$, $L_{2}, L_{3}, C_{1}, C_{2}, C_{3}$, and $C_{4}$ ) are employed for the input matching network with a $Q_{n}$ of within 1.6 to widen $3-\mathrm{dB}$ gain bandwidth of the stacked PAs. The dc current source is designed as the bias circuit at the base terminal of HBT/CE transistor to stabilize the dc bias [23].

The chip photos of the Type-I and Type-II triple-stacked PAs are shown in Fig. 8. The chip sizes are $1.6 \times 1 \mathrm{~mm}^{2}$, and $1.5 \times 1 \mathrm{~mm}^{2}$ for the Type-I and the Type-II triple-stacked PAs, respectively. The transistors are biased at saturation region for HEMT and active region for HBT. The RF performances are measured via on-wafer probing. The simulated and measured large-signal gains with an input power of $5 \mathrm{dBm}$ are shown in Fig. 9 for the Type-I and the Type-II triple-stacked PAs. The measured large-signal gain of the Type-I triplestacked PA for $V_{g 2}=3.6$ and $V_{g 3}=10 \mathrm{~V}$ is higher than $10 \mathrm{~dB}$ from 3 to $6 \mathrm{GHz}$. The measured large-signal gain of the Type-II triple-stacked PA for $V_{g 2}=3.6$ and $V_{g 3}=15 \mathrm{~V}$ is higher than $10 \mathrm{~dB}$ from 3.5 to $5 \mathrm{GHz}$. The simulated and

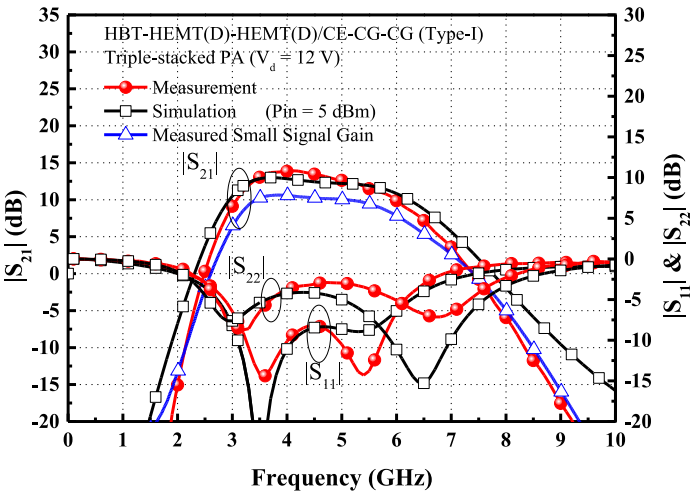

(a)

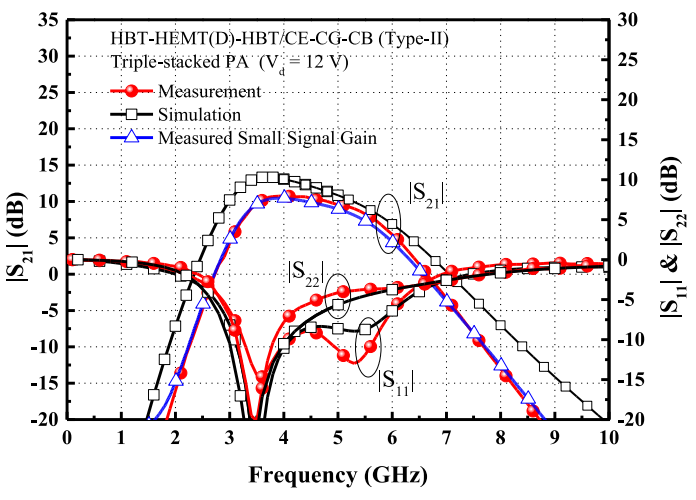

(b)

Fig. 9 Simulated and measured large signal gains, input and output return losses of the triple-stacked PAs, (a) Type-I, and (b) Type-II, where the input power (Pin) is $5 \mathrm{dBm}$.

measured output powers, and PAEs versus input power with a dc supply voltage $V_{d d}$ of $12 \mathrm{~V}$ are shown in Fig. 10 for the Type-I and the Type-II triple-stacked PAs. The measured output $1-\mathrm{dB}$ compression points $\left(\mathrm{P}_{1 \mathrm{~dB}}\right)$ are $29.6 \mathrm{dBm}$ and $29.7 \mathrm{dBm}$ for the Type-I PA and the Type-II triplestacked PAs, respectively. The measured maximum PAE for the Type-I and the Type-II triple-stacked PAs is $38.3 \%$ and $30.6 \%$, respectively. Both of the proposed PAs can achieve the maximum output power of higher than $30 \mathrm{dBm}$. The proposed Type-I triple-stacked PA has wider bandwidth and higher maximum output power than the Type-II configuration. The experimental results agree well with the discussion in Section 2. The linearity characteristics of the 
Table II Performance comparison of the previously reported stacked power amplifiers and this work.

\begin{tabular}{|c|c|c|c|c|c|c|c|c|c|c|c|}
\hline Ref. & Topology & DC (V) & $\begin{array}{l}\text { Freq. } \\
\text { (GHz) }\end{array}$ & $\begin{array}{c}\text { Bandwidth } \\
(\%)\end{array}$ & $\begin{array}{l}\text { Gain } \\
\text { (dB) }\end{array}$ & $\begin{array}{c}\mathbf{P}_{1 \mathrm{~dB}} \\
(\mathrm{dBm})\end{array}$ & $\begin{array}{c}P_{\text {sat }} \\
(\mathbf{d B m})\end{array}$ & $\begin{array}{l}\text { PAE (\%) } \\
\left(\mathbf{P}_{1 \mathrm{~dB}} / \mathbf{P}_{\text {sat }}\right)\end{array}$ & FOM & $\begin{array}{c}\text { Fully } \\
\text { Integrated }\end{array}$ & Process \\
\hline \multirow[t]{2}{*}[14]{} & 2-stage & $\begin{array}{c}2.4 \\
\text { (driver) } \\
\end{array}$ & 47 & - & 24.9 & - & 20.1 & $-/ 15.6$ & 70.4 & Yes & \multirow{2}{*}{$\begin{array}{c}45 \mathrm{~nm} \text { SOI } \\
\text { CMOS }\end{array}$} \\
\hline & 4-stacked & 4.8 & 47.5 & 40 & 12.8 & - & 20.3 & $-/ 19.4$ & 59.5 & Yes & \\
\hline$[16]$ & 2-stacked & 6 & $0.1-6.5$ & 193 & $\begin{array}{r}18.4 \\
\pm 1.5 \\
\end{array}$ & $18-19$ & 22 & $-/ 17$ & 48.3 & Yes & $\begin{array}{c}0.18 \mu \mathrm{m} \\
\text { CMOS process }\end{array}$ \\
\hline$[17]$ & 3-stacked & 2.8 & $25-30.5$ & 20 & 9.9 & 14.9 & 18.8 & $-/ 23.4$ & 31.3 & & $\begin{array}{c}22 \mathrm{~nm} \text { SOI } \\
\text { CMOS }\end{array}$ \\
\hline$[18]$ & 3-stack & 3.6 & 38 & - & 17.5 & 14.8 & 17.3 & $-/ 24.3$ & 39.8 & Yes & $65 \mathrm{~nm} \mathrm{CMOS}$ \\
\hline [19] & $\begin{array}{l}\text { 4-parallel } \\
\text { 3-stacked } \\
\end{array}$ & 15 & $4-10$ & 85.7 & $\begin{array}{r}13.5 \\
\pm 1.5 \\
\end{array}$ & - & $35-37$ & $-/ 25-32$ & 67.6 & Yes & $\begin{array}{c}0.15 \mu \mathrm{m} \text { GaAs } \\
\text { HEMT }\end{array}$ \\
\hline$[23]$ & $\begin{array}{c}\text { 2-stacked } \\
\text { CE-CG }\end{array}$ & $\begin{array}{c}8 \\
12\end{array}$ & $\begin{array}{l}3.5-6.5 \\
3.5-6.5\end{array}$ & $\begin{array}{l}60 \\
60\end{array}$ & $\begin{array}{c}13.5 \\
11\end{array}$ & $\begin{array}{l}26.4 \\
29.1\end{array}$ & $\begin{array}{c}27 \\
29.4\end{array}$ & $\begin{array}{c}35.6 / 38 \\
28.5 / 27.2\end{array}$ & $\begin{array}{l}50.3 \\
48.7\end{array}$ & Yes & $\begin{array}{c}2 \mu \mathrm{m} / 0.5 \mu \mathrm{m} \\
\text { GaAs HBT- } \\
\text { HEMT }\end{array}$ \\
\hline \multirow{2}{*}{$\begin{array}{l}\text { This } \\
\text { work }\end{array}$} & $\begin{array}{c}\text { 3-stacked } \\
\text { CE-CG-CG }\end{array}$ & 12 & $3.1-5.8$ & 60.6 & $\begin{array}{l}15.7 \\
9.9^{*}\end{array}$ & 29.57 & 31.67 & $28.8 / 38.3$ & $\begin{array}{c}57.2 \\
51.4^{*} \\
\end{array}$ & Yes & \multirow{2}{*}{$\begin{array}{c}2 \mu \mathrm{m} / 0.5 \mu \mathrm{m} \\
\text { GaAs HBT- } \\
\text { HEMT }\end{array}$} \\
\hline & $\begin{array}{c}\text { 3-stacked } \\
\text { CE-CG-CB } \\
\end{array}$ & 12 & $3.3-5.6$ & 51.7 & $\begin{array}{l}14.9 \\
8.4^{*} \\
\end{array}$ & 29.7 & 30.5 & $30.5 / 30.6$ & $\begin{array}{c}54.2 \\
47.8^{*} \\
\end{array}$ & Yes & \\
\hline
\end{tabular}

*small signal gain

triple-stack PAs are measured using continuous-wave (CW) two-tone signals with a frequency spacing of $2 \mathrm{MHz}$, and the central frequency is $5 \mathrm{GHz}$. The measured third-order IMDs (IMD3s) of Type-I and Type-II PAs are $30 \mathrm{dBc}$ with an input power of $8.5 \mathrm{dBm}$. To evaluate the circuit performance of the previously reported stacked PAs and this work, a figure-of-merit (FOM) is defined as [14]

$$
\mathrm{FOM}=\mathrm{P}_{\text {sat }}+\mathrm{G}+10 \log _{10} \mathrm{PAE}+20 \log _{10} f_{0}
$$

where $P_{\text {sat }}$ is the saturated output power in $\mathrm{dBm}, \mathrm{G}$ is the power gain in $\mathrm{dB}$, and $f_{0}$ is the center frequency in $\mathrm{GHz}$. Table II shows the performance comparison of the previously reported stacked PAs and this work. Our proposed triplestacked PAs perform high maximum output power with good PAE. As compared the bandwidth to [16], the bandwidth of the PA in [16] is wider than the proposed PAs, due to the used of external bias-tee network. Although the bias-tee achieves broadband frequency response at the output, the cost of the PA increases. As compared to the PA in [23], the proposed triple-stacked PAs have better PAEs at output $\mathrm{P}_{1 \mathrm{~dB}}$, and better output saturated power $\left(\mathrm{P}_{\mathrm{sat}}\right)$ than the dualstacked PA with a dc supply voltage $V_{d d}$ of $12 \mathrm{~V}$. With the HEMT(D)/CG as the third-stacked transistor, the maximum output power is enhanced with good PAE. The CE-CG-CG configuration triple-stacked PA also has the same bandwidth as the CE-CG compared to the dual-stacked PA in [23].

\section{Conclusion}

Two monolithic triple-stacked PAs using a $2-\mu \mathrm{m} / 0.5-\mu \mathrm{m}$ GaAs HBT-HEMT process are successfully presented in this paper. Based on a CE-CG configuration with stacked $\mathrm{CG}$ and $\mathrm{CB}$ configurations, the proposed triple-stacked PAs demonstrate high output power and broad bandwidth. The proposed CE-CG-CG triple-stacked PA has wider bandwidth than the CE-CG-CB configuration. It can be further utilized for the modern mobile applications due to its good circuit performance and the mass-production MMIC process.

\section{Acknowledgments}

This work was supported in part by the Ministry of Science and Technology (MOST), Taiwan, under Grants MOST 106-2628-E-008-001-MY3 and MOST 108-2731-M-008001. The chips were fabricated by the WIN Semiconductors Corporation, Taoyuan, Taiwan. The EDA design software is provided by the Taiwan Semiconductor Research Institute (TSRI), Taiwan. The authors would like to thank Prof. G. D. Vendelin, National Central University, Jhongli City, Taiwan, for the discussions and the measurement helps.

\section{References}

[1] A.K. Ezzeddine, et al:: "The high voltage/high power FET (HiVP)," IEEE RFIC Symp. Digest (2003) 215 (DOI: 10.1109/RFIC.2003. 1213929).

[2] Z.-M. Tsai, et al.: "An HBT four-cell monolithic stacked power amplifier," IEEE MTTS Int. Microw. Symp. Digest (2007) 151 (DOI: 10.1109/MWSYM.2007.380312).

[3] C.-C. Shen, et al.: "A broadband stacked power amplifier using 2$\mu \mathrm{m}$ GaAs HBT process for C-band applications," 2008 Asia Pacific Microw. Conf. Proc. (2008) (DOI: 10.1109/APMC.2008.4958388).

[4] D. Fritsche, et al.: "Analysis and design of a stacked power amplifier with very high bandwidth," IEEE Trans. Microw. Theory Techn. 60 (2012) 3223 (DOI: 10.1109/TMTT.2012.2209439).

[5] K. Datta, et al.: "A triple-stacked class-E mm-wave SiGe HBT power amplifier," IEEE MTTS Int. Microw. Symp. Digest (2013) 151 (DOI: 10.1109/MWSYM.2013.6697457).

[6] C. Liu, et al.: "An $890 \mathrm{~mW}$ stacked power amplifier using SiGe HBTs for X-band multifunctional chips," 41st ESSCIRC (2015) (DOI: 10.1109/ESSCIRC.2015.7313830).

[7] M. Squartecchia, et al.: "Design procedure for millimeter-wave InP DHBT stacked power amplifiers," 2015 INMMiC (2015) (DOI: 10.1109/INMMIC.2015.7330368).

[8] K. Datta, et al.: "Performance limits, design and implementation of mm-wave SiGe HBT class-E and stacked class-E power amplifiers," IEEE J. Solid-State Circuit 49 (2014) 2150 (DOI: 10.1109/ JSSC.2014.2353800).

[9] S.M.A. Ali, et al.: "A 38-GHz millimeter-wave double-stacked HBT class- $\mathrm{F}^{-1}$ high-gain power amplifier in 130-nm SiGe-BiCMOS," IEEE Trans. Microw. Theory Techn. (2020) (DOI: 10.1109/TMTT. 2020.2988874)

[10] K.W. Kobayashi, et al.: "Baseband to $140-\mathrm{GHz}$ SiGe HBT and 100GHz InP DHBT broadband triple-stacked distributed amplifiers with active bias terminations," IEEE J. Solid-State Circuit (2020) (DOI: 
10.1109/JSSC.2020.2988420).

[11] J.-H. Chen, et al.: "A broadband stacked power amplifier in 45-nm CMOS SOI technology," IEEE J. Solid-State Circuits 48 (2013) 2775 (DOI: 10.1109/JSSC.2013.2276135).

[12] J.-H. Chen, et al.: "A wideband power amplifier in $45 \mathrm{~nm}$ CMOS SOI technology for X band applications," IEEE Microw. Compon. Lett. 23 (2013) 587 (DOI: 10.1109/LMWC.2013.2279117).

[13] H.-T. Dabag, et al.: "Analysis and design of stacked-FET millimeterwave power amplifiers," IEEE Trans. Microw. Theory Techn. 61 (2013) 1543 (DOI: 10.1109/TMTT.2013.2247698).

[14] A. Chakrabarti, et al.: "High-power high-efficiency class-E-like stacked mmWave PAs in SOI and Bulk CMOS: theory and implementation," IEEE Trans. Microw. Theory Techn. 62 (2014) 1686 (DOI: 10.1109/TMTT.2014.2327919).

[15] M. Fathi, et al.: "A stacked 6.5-GHz 29.6-dBm power amplifier in standard 65-nm CMOS," Proc. IEEE Custom Integr. Circuits Conf. (2010) 1 (DOI: 10.1109/CICC.2010.5617403).

[16] H.-F. Wu, et al.: "Analysis and design of an ultrabroadband stacked power amplifier in CMOS technology," IEEE Trans. Circuits Syst. II, Exp. Briefs 63 (2016) 49 (DOI: 10.1109/TCSII.2015.2504926).

[17] J.P. Aikio, et al.: "Ka-Band 3-stack power amplifier with $18.8 \mathrm{dBm}$ Psat and $23.4 \%$ PAE using 22nm CMOS FDSOI technology," IEEE Topical Conf. RF/Microw. Power Amp. Radio Wireless (2019) (DOI: 10.1109/PAWR.2019.8708719).

[18] Y. Chang, et al.: "A Ka-band stacked power amplifier with 24.8$\mathrm{dBm}$ output power and 24.3\% PAE in 65-nm CMOS technology,” IEEE MTT-S Inter. Microw. Symp. Dig. (2019) 316 (DOI: 10.1109/MWSYM.2019.8700937).

[19] H. Wu, et al.: "A 4-10 GHz fully-integrated stacked GaAs pHEMT power amplifier," IEEE MTT-S Inter. Microw. Symp. (2017) 24 (DOI: 10.1109/MWSYM.2017.8059091).

[20] C. Lee, et al:: "A $18 \mathrm{GHz}$ broadband stacked FET power amplifier using $130 \mathrm{~nm}$ metamorphic HEMTs," IEEE Microw. Compon. Lett. 19 (2009) 828 (DOI: 10.1109/LMWC.2009.2033533).

[21] M. Gavell, et al.: "A high voltage mm-wave stacked HEMT power amplifier in $0.1 \mu \mathrm{m}$ InGaAs technology," IEEE MTT-S Inter. Microw. Symp. (2015) 1 (DOI: 10.1109/MWSYM.2015.7166754).

[22] F. Thome, et al.: "A 50-nm gate-length metamorphic HEMT distributed power amplifier MMIC based on stacked-HEMT unit cells," IEEE MTT-S Inter. Microw. Symp. (2017) 1695 (DOI: 10.1109/ MWSYM.2017.8058967).

[23] C.-C. Shen, et al.: "A monolithic 3.5-to-6.5 GHz GaAs HBTHEMT/common-emitter and common-gate stacked power amplifier," IEEE Microw. Compon. Lett. 22 (2012) 474 (DOI: 10.1109/ LMWC.2012.2210034).

[24] G.-Y. Chen, et al.: "Dseign and analysis of a Ka-band monolithic high efficiency frequency quadrupler using GaAs HBT-HEMT commonbase/common-source balance topology," IEEE Trans. Microw. Theory Techn. 61 (2013) 3674 (DOI: 10.1109/TMTT.2013.2277991).

[25] H.-Y. Chang, et al.: "Design and analysis of a DC-43.5-GHz fully integrated distributed amplifier using GaAs HEMT-HBT cascade gain stage," IEEE Trans. Microw. Theory Techn. 59 (2011) 443 (DOI: 10.1109/TMTT.2010.2092786).

[26] S.-H. Chen, et al.: "A monolithic DC-31 GHz distributed amplifier using cascode HBT-NMOS gain cell in $0.18 \mu \mathrm{m}$ SiGe technology," APMC Dig. (2012) 211 (DOI: 10.1109/APMC.2012.6421549).

[27] G.-Y. Chen, et al.: "2.8 dB conversion gain broadband HBT-HEMT balanced frequency tripler with high harmonic suppression," Electron. Lett. 50 (2014) 812 (DOI: 10.1049/el.2014.0678).

[28] C.-H. Lu, et al.: "Monolithic 2- $\mu \mathrm{m} / 0.5-\mu \mathrm{m}$ GaAs HBT-HEMT (BiHEMT) process for low phase noise voltage controlled oscillators (VCOs)," APMC Dig. (2012) 1235 (DOI: 10.1109/APMC.2012. 421880).

[29] X. Xia, et al:: "An improved colpitts VCO with low phase noise using a GaAs BiHEMT process," IEEE Microw. Compon. Lett. 30 (2020) 70 (DOI: 10.1109/LMWC.2019.2953578).

[30] K.W. Kobayashi, et al.: "A monolithically integrated HEMT-HBT low noise high linearity variable gain amplifier," IEEE J. Solid-State Circuit 31 (1996) 714 (DOI: 10.1109/4.509854). 